\title{
Post-stroke Hyperglycemia in Non-diabetic Ischemic Stroke is Related With Worse Functional Outcome: A Cohort Study
}

\author{
Jin A Yoon, MD", Yong-Il Shin, MD, PhD², Deog Young Kim, MD, PhD ${ }^{3}$, Min Kyun Sohn, MD, $\mathrm{PhD}^{4}$, \\ Jongmin Lee, $\mathrm{MD}, \mathrm{PhD}^{5}$, Sam-Gyu Lee, $\mathrm{MD}, \mathrm{PhD}^{6}$, Yang-Soo Lee, $\mathrm{MD}, \mathrm{PhD}^{7}$, Eun Young Han, $\mathrm{MD} \mathrm{PhD}^{8}$, \\ Min Cheol Joo, MD, $\mathrm{PhD}^{9}$, Gyung-Jae Oh, MD, $\mathrm{PhD}^{9}$, Minsu Park, $\mathrm{MS}^{1}$, Won Hyuk Chang, MD, $\mathrm{PhD}^{10}$, \\ Yun-Hee Kim, MD, PhD $^{10}$
}

${ }^{1}$ Department of Rehabilitation Medicine, Pusan National University School of Medicine and Biomedical Research Institute, Pusan National University Hospital, Busan;

${ }^{2}$ Department of Rehabilitation Medicine, Pusan National University Yangsan Hospital, Pusan National University College of Medicine, Yangsan;

${ }^{3}$ Department and Research Institute of Rehabilitation Medicine, Yonsei University College of Medicine, Seoul; ${ }^{4}$ Department of Rehabilitation Medicine, Chungnam National University School of Medicine, Daejeon;

${ }^{5}$ Department of Rehabilitation Medicine, Konkuk University School of Medicine, Seoul;

${ }^{6}$ Department of Physical and Rehabilitation Medicine, Chonnam National University Medical School, Gwangju;

${ }^{7}$ Department of Rehabilitation Medicine, Kyungpook National University College of Medicine, Daegu;

${ }^{8}$ Department of Rehabilitation Medicine, Jeju National University Hospital, Jeju University College of Medicine, Jeju;

${ }^{9}$ Department of Rehabilitation Medicine, Wonkwang University School of Medicine, Iksan;

${ }^{10}$ Department of Physical and Rehabilitation Medicine, Center for Prevention and Rehabilitation, Heart Vascular and Stroke Institute, Samsung Medical Center, Sungkyunkwan University School of Medicine, Seoul, Korea

\footnotetext{
Received July 2, 2021; Revised July 30, 2021; Accepted Augsut 23, 2021; Published online October 31, 2021 Corresponding author: Yong-Il Shin

Department of Rehabilitation Medicine, Pusan National University Yangsan Hospital, Pusan National University School of Medicine, 20, Geumo-ro, Mulgeum-eu, Yangsan 626-770, Korea. Tel: +82-55-360-4250, Fax: +82-55-360-4251, E-mail: rmshin@pusan.ac.kr

Co-corresponding author: Yun-Hee Kim

Department of Physical and Rehabilitation Medicine, Center for Prevention and Rehabilitation, Heart Vascular and Stroke Institute, Samsung Medical Center, Sungkyunkwan University School of Medicine, 50 Ilwon-dong, Gangnam-gu, Seoul 135-710, Korea. Tel: +82-2-3410-2824, Fax: +82-2-34100052, E-mail: yunkim@skku.edu

ORCID: Jin A Yoon (https://orcid.org/0000-0001-5762-0559); Yong-Il Shin (https://orcid.org/0000-0001-7894-0930); Deog Young Kim (https://orcid. org/0000-0001-7622-6311); Min Kyun Sohn (https://orcid.org/0000-0002-2548-545X); Jongmin Lee (https://orcid.org/0000-0001-8718-0099); Sam-Gyu Lee (https://orcid.org/0000-0002-3059-3981); Yang-Soo Lee (https://orcid.org/0000-0002-8855-1231); Eun Young Han (https://orcid.org/0000-00024780-8862); Min Cheol Joo (https://orcid.org/0000-0003-2778-4194); Gyung-Jae Oh (https://orcid.org/0000-0001-9869-1564); Minsu Park (https:// orcid.org/0000-0002-3995-4122); Won Hyuk Chang (https://orcid.org/0000-0002-4969-7895); Yun-Hee Kim (https://orcid.org/0000-0001-6101-8851).
}

@) This is an open-access article distributed under the terms of the Creative Commons Attribution Non-Commercial License (http://creativecommons.org/ licenses/by-nc/4.0) which permits unrestricted noncommercial use, distribution, and reproduction in any medium, provided the original work is properly cited. Copyright ( 2021 by Korean Academy of Rehabilitation Medicine 
Objective To investigate long-term and serial functional outcomes in ischemic stroke patients without diabetes with post-stroke hyperglycemia.

Methods The Korean Stroke Cohort for Functioning and Rehabilitation (KOSCO) is a large, multi-center, prospective cohort study of stroke patients admitted to participating hospitals in nine areas of Korea. From KOSCO, ischemic stroke patients without diabetes were recruited and divided into two groups: patients without diabetes without $(n=779)$ and with post-stroke hyperglycemia $(n=223)$. Post-stroke hyperglycemia was defined as a glucose level $>8 \mathrm{mmol} / \mathrm{L}$. Functional assessments were performed 7 days and 3, 6, and 12 months after stroke onset.

Results There were no significant differences in baseline characteristics between the groups, except in the age of onset and smoking. Analysis of the linear correlation between the initial National Institutes of Health Stroke Scale (NIHSS) score and glucose level showed no significant difference. Among our functional assessments, NIHSS, Fugl-Meyer Assessment (affected side), Functional Ambulatory Category, modified Rankin Scale, and Korean Mini-Mental State Examination (K-MMSE) showed statistically significant improvements in each group. All functional improvements except K-MMSE were significantly higher in patients without post-stroke hyperglycemia at 7 days and 3, 6, and 12 months.

Conclusion The glucose level of ischemic stroke patients without diabetes had no significant correlation with the initial NIHSS score. The long-term effects of stress hyperglycemia showed worse functional outcomes in ischemic stroke patients without diabetes with post-stroke hyperglycemia.

Keywords Cohort studies, Recovery of function, Ischemic stroke, Hyperglycemia

\section{INTRODUCTION}

Diabetes is a well-known independent predictor of stroke [1], and the risk of stroke cannot be reduced even in patients with diabetes with well-controlled glucose levels. Excluding patients who are unaware of their diabetes and are not receiving treatment, approximately $20 \%-50 \%$ of acute ischemic stroke patients are hyperglycemic, despite the absence of pre-diagnosed diabetes or impaired glucose tolerance (IGT) [2-4]. An increase in counterregulatory hormones such as glucagon, epinephrine, norepinephrine, and growth hormone enhances hepatic gluconeogenesis and decreases peripheral glucose uptake, thus inducing a hyperglycemic state [5]. Increased cytokine levels in response to stress-related reactions are also considered to cause abnormal increases in the serum glucose levels in acute stroke patents [6].

In human and animal studies, an association was found between stress-induced hyperglycemia and increased cerebral lactate levels, resulting in brain tissue acidosis [7]. Hyperglycemia also adversely affects the ischemic brain by disrupting the blood-brain barrier and accelerating cerebral edema [4], which is associated with impaired cerebrovascular reactivity in the microvasculature upon reperfusion therapy [3]. This phenomenon is known to increase the cerebral infarct size and consequently worsen neurologic outcomes. Magnetic resonance imaging (MRI) was used to demonstrate the loss of penumbra tissue upon elevated glucose levels [8]. Two meta-analyses showed that even mildly elevated glucose levels at admission were associated with a three-fold higher risk of stroke- and myocardial infarction-related mortality, indicating that post-stroke hyperglycemia is a poor prognostic indicator $[9,10]$. The glucose level should be strictly controlled with insulin upon admission to the emergency room in stroke patients since insulin acts directly on the brain parenchyma, which reduces neuronal necrosis in the cortex, striatum, and hippocampus [11,12].

Intensive rehabilitation can enhance functional improvements after hemorrhagic stroke as patients with stress hyperglycemia had lower scores on all functional assessments during all time points among patients with subarachnoid hemorrhage (SAH) [13]. The long-term functional outcomes of patients with ischemic stroke have not yet been established. A prospective cohort study, in conjunction with the Korean Stroke Cohort for Functioning and Rehabilitation (KOSCO), was conducted to investigate the long-term and serial functional out- 
comes of ischemic stroke patients without diabetes and with post-stroke hyperglycemia.

\section{MATERIALS AND METHODS}

\section{Study design}

KOSCO is a large, multi-center, prospective cohort study of all first-time acute stroke patients admitted to participating hospitals in nine distinct areas of Korea. This 10-year longitudinal follow-up study investigates the residual effects, activity limitations, and quality of life of first-time stroke patients.

\section{Ethics statement}

The research was approved by the Research Ethics Committee of Pusan National University Yangsan Hospital (No. 05-2012-057). Written informed consent was obtained from all patients prior to the study. If the patient was unable to provide consent, it was obtained from the patient's legal representative.

\section{Study subjects}

All patients with first-time acute stroke admitted to the study hospitals were asked to participate. The inclusion criteria were: (1) first-time acute ischemic stroke, including supratentorial and infratentorial lesions, with corresponding evidence of acute arterial occlusion on computed tomography/CTA (computed tomography angiography) or MRI/MRA (magnetic resonance angiography); (2) age $\geq 19$ years at stroke onset. Acute stroke was defined as a rapidly evolving, focal neurological deficit persisting for $>24$ hours. The exclusion criteria were: (1) recurrent stroke, (2) history of transient ischemic attack, (3) traumatic/non-traumatic intracerebral hemorrhage, (4) a diagnosis of diabetes before admission, (5) uncontrolled diabetes before stroke onset-glycated hemoglobin (HbAlc) level ( $\geq 6.2 \%$ ) [14]; (6) unknown medical history; (7) other associated major illnesses such as myocardial infarction; and (8) pulmonary embolism or active infection as confirmed by radiologic or laboratory studies.

\section{Procedure}

All eligible patients were recruited at the time of stroke evaluation. Patient enrollment was planned over a 3-4year period. The patients were enrolled in the study after written informed consent was obtained from the patients or their legal representatives.

\section{Baseline review of medical records}

A complete enumeration survey of all patients was performed for the baseline using a review of the medical records on the first admission. The presence of cerebrovascular risk factors was assessed using standardized, structured questionnaires and classified according to the current guidelines of the American Heart Association [15]. Comorbidities were assessed using the Charlson Comorbidity Index [16]. Initial stroke severity was recorded at the time of hospital arrival using the National Institutes of Health Stroke Scale (NIHSS) for ischemic strokes [17].

Neuroimaging was performed on all patients; CTA, MRA, or ultrasonography according to the standards of clinical practices. Etiology was based on neuroimaging, medical history, and medication use; the TOAST (Trial of ORG 10172 in Acute Stroke Treatment) classification was used to categorize the etiologies [18].

Post-stroke hyperglycemia was defined as a randomly tested glucose level $>8 \mathrm{mmol} / \mathrm{L}$ ( $144 \mathrm{mg} / \mathrm{dL}$ ) upon admission $[9,19]$. The HbAlc levels were assessed to rule out previously undiagnosed diabetes and IGT. In total, 1,002 patients fulfilled the criteria based on the baseline data gathered in August 2012. These patients were divided into two groups: patients without diabetes without $(n=779)$ and with stress hyperglycemia $(\mathrm{n}=223)$.

\section{Follow-up}

A two-step approach was used on all patients for followup assessment. First, they were contacted by telephone. If this approach failed, a second telephone call was made to contact the patients' caregivers. Information on the patients' status was obtained through these telephone interviews. All living patients were invited to visit the research center for functional assessments, structured self-administered questionnaires, and face-to-face interviews. If the patients were unable to visit the research center, the investigations were performed in their homes. The primary caregivers visited our researchers for structured self-administered questionnaires and face-to-face interviews.

\section{Functional assessments \\ Baseline}


Baseline evaluation was performed 7 days after stroke onset using face-to-face functional assessments, including the Korean version of NIHSS (K-NIHSS) for stroke severity [17], Korean Mini-Mental State Examination (K-MMSE) for cognitive function [20], Fugl-Meyer Assessment (FMA) for motor function [21], Functional Ambulatory Category (FAC) for mobility and gait [22], and the modified Rankin Scale (mRS) for general functional assessment [23].

\section{Follow-up}

All patients were evaluated using face-to-face functional assessments, which were performed at 3,6 , and 12 months post-discharge. The measures of outcomes, baseline, and Functional Independence Measure (FIM) utilized the same batteries [24], and a Korean version of the modified Barthel Index (K-MBI) [25] were added for activities of daily living (ADL) assessment. All functional assessments were conducted as blind evaluations by the same occupational therapist.

\section{Statistical analysis}

Statistical analysis was performed using SPSS for Windows version 21.0 (IBM, Armonk, NY, USA). Descriptive statistics and frequency, crosstabs, and chi-square distribution analyses were used to compare data obtained from the baseline review of medical records and initial stroke characterization. Scale factors were analyzed using descriptive statistics and an independent t-test. Oneway ANOVA was used to analyze the functional outcome variation over time in each group. Generalized estimating equations (GEE) were used after correcting for age, sex, and initial NIHSS score to find the intergroup difference of functional improvement by stress hyperglycemia. $\mathrm{p}<0.05$ was considered significant.

\section{RESULTS}

Initially, 1,084 patients enrolled in our study, with a final sample size of 1,002 patients. The follow-up rate was $7.7 \%(n=84)$ due to incomplete follow up. Among the 1,002 patients, $779(78 \%)$ and $223(22 \%)$ had an initial serum glucose level less than and greater than $8 \mathrm{mmol} / \mathrm{L}$ (144 mg/dL), respectively (Fig. 1).

On comparing the baseline characteristics, age of onset and smoking habits showed significant differences between the groups $(\mathrm{p}<0.05)$. There were no significant differences in the body mass index; waist circumference; and stroke risk factors such as hypertension, atrial fibrillation, hyperlipidemia, family history, and alcohol consumption history. There was also no significant difference in the types of ischemic stroke, initial NIHSS scores, and hospital arrival time between the groups (Table 1).

The degree of serial improvements in extremity functioning and the performance of ADLs were compared. Among our functional assessments, NIHSS, FMA (affected side), FAC, mRS, and K-MMSE, representing stroke severity and motor and cognitive function, showed statistically significant improvements at 7 days and 3, 6, and 12 months in the two groups $(\mathrm{p}<0.05)$.

All functional assessments, excluding K-MMSE, showed worse scores in patients with post-stroke hyperglycemia than in patients without post-stroke hyperglycemia at 7 days and 3, 6, and 12 months $(\mathrm{p}<0.05)$. The NIHSS score

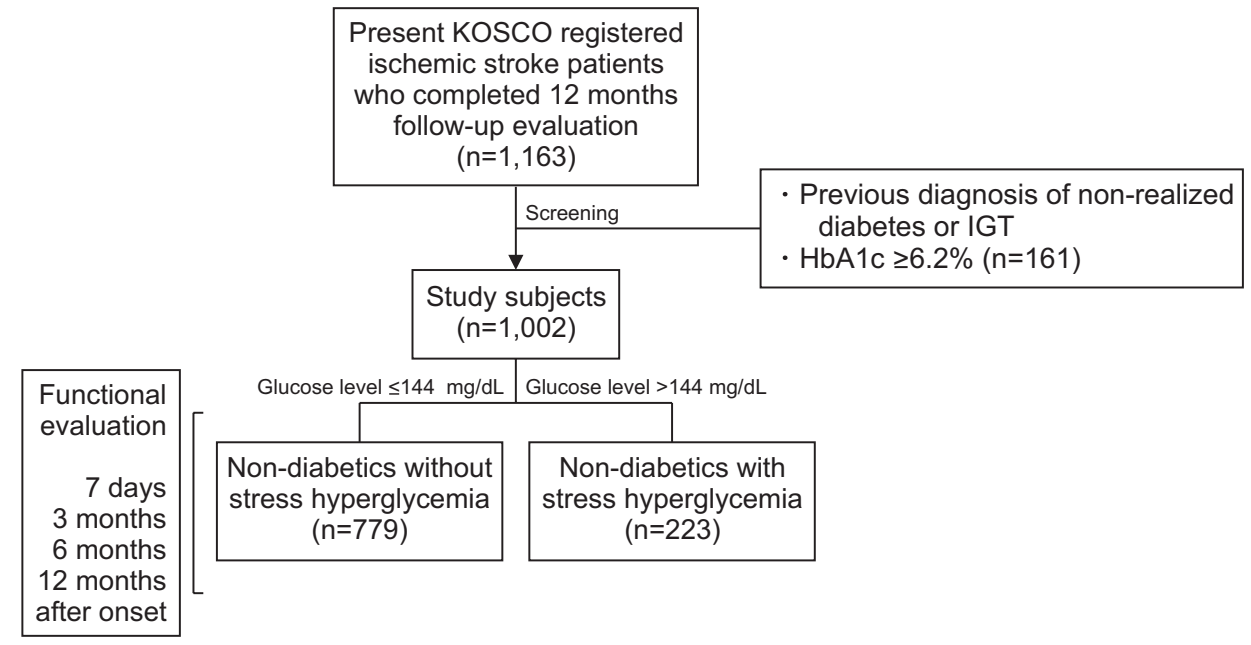

Fig. 1. Flow diagram of this study. KOSCO, Korean Stroke Cohort for Functioning and Rehabilitation; IGT, impaired glucose tolerance; HbAlc, glycated hemoglobin. 
Table 1. Baseline characteristics according to stress hyperglycemia of stroke patients

\begin{tabular}{|c|c|c|c|c|}
\hline Parameter & $\begin{array}{c}\text { Total } \\
(\mathrm{n}=1,002)\end{array}$ & $\begin{array}{c}\text { Non-diabetics } \\
\text { without post-stroke } \\
\text { hyperglycemia }(n=779)\end{array}$ & $\begin{array}{c}\text { Non-diabetics } \\
\text { with post-stroke } \\
\text { hyperglycemia }(n=223)\end{array}$ & p-value \\
\hline Sex, male & $606(60.5)$ & $481(61.7)$ & $125(56.1)$ & $0.125^{\mathrm{a})}$ \\
\hline Age (yr) & $67.51 \pm 13.04$ & $67.07 \pm 13.38$ & $69.02 \pm 11.65$ & $0.034^{\mathrm{c})}$ \\
\hline $\operatorname{BMI}\left(\mathrm{kg} / \mathrm{m}^{2}\right)$ & $23.42 \pm 3.00$ & $23.38 \pm 2.94$ & $23.55 \pm 3.24$ & $0.471^{c)}$ \\
\hline Waist circumference $(\mathrm{cm})$ & $82.83 \pm 8.69$ & $82.69 \pm 8.40$ & $83.42 \pm 9.88$ & $0.485^{\mathrm{c})}$ \\
\hline \multicolumn{5}{|l|}{ Risk factors of stroke } \\
\hline Hypertension & $504(50.3)$ & $386(49.6)$ & $118(52.9)$ & $0.376^{\text {a) }}$ \\
\hline Coronary heart disease & $36(3.6)$ & $35(4.5)$ & $4(1.8)$ & $0.167^{\mathrm{a})}$ \\
\hline Atrial fibrillation & $84(8.4)$ & $82(10.6)$ & $22(9.9)$ & $0.457^{\mathrm{a})}$ \\
\hline Hyperlipidemia & $129(12.9)$ & $99(12.7)$ & $30(13.5)$ & $0.770^{\mathrm{a})}$ \\
\hline Unruptured intracranial aneurysm & $5(0.5)$ & $5(0.6)$ & $0(0.0)$ & $0.593^{\mathrm{b})}$ \\
\hline Obesity & $97(9.7)$ & $78(10.0)$ & $19(8.5)$ & $0.607^{\mathrm{a})}$ \\
\hline Family history & $79(7.9)$ & $66(8.5)$ & $13(5.8)$ & $0.197^{\mathrm{a})}$ \\
\hline Smoking & & & & $0.040^{\mathrm{a})}$ \\
\hline Current smokers & $297(29.6)$ & $244(31.3)$ & $53(23.8)$ & \\
\hline Former smokers & $113(11.3)$ & $91(11.7)$ & $22(9.9)$ & \\
\hline Never smokers & $592(59.1)$ & $444(57.0)$ & $148(66.4)$ & \\
\hline Alcohol consumption & & & & $0.242^{\mathrm{a})}$ \\
\hline None & $625(62.4)$ & $496(63.7)$ & $129(57.8)$ & \\
\hline Moderate & $256(25.5)$ & $190(24.4)$ & $66(29.6)$ & \\
\hline Heavy & $121(12.1)$ & $93(11.9)$ & $28(12.6)$ & \\
\hline Ischemic type (TOAST) & & & & $0.307^{\mathrm{a})}$ \\
\hline Large-artery atherosclerosis & $472(47.1)$ & $374(48.0)$ & $98(43.9)$ & \\
\hline Small-artery occlusion & $196(19.6)$ & $158(20.3)$ & $38(17.0)$ & \\
\hline Cardioembolism & $149(14.9)$ & $110(14.1)$ & $39(17.5)$ & \\
\hline Stroke of other determined etiology & $73(7.3)$ & $56(7.2)$ & $17(7.6)$ & \\
\hline Stroke of undetermined etiology & $112(11.2)$ & $81(10.4)$ & $31(13.9)$ & \\
\hline Ischemic location & & & & $0.900^{\mathrm{c})}$ \\
\hline Supratentorial lesion & $932(93.0)$ & $725(93.1)$ & $207(92.8)$ & \\
\hline Infratentorial lesion & $70(7.0)$ & $54(6.9)$ & $16(7.2)$ & \\
\hline Hospital arrival time after onset (hr) & $4.02 \pm 4.39$ & $4.00 \pm 4.47$ & $4.10 \pm 4.13$ & $0.811^{\mathrm{c})}$ \\
\hline Initial NIHSS score & $5.29 \pm 5.73$ & $5.17 \pm 5.56$ & $5.71 \pm 6.31$ & $0.211^{\mathrm{c})}$ \\
\hline \multicolumn{5}{|l|}{ Initial blood parameter } \\
\hline Glucose (mg/dL) & $128.06 \pm 38.1$ & $113.08 \pm 15.60$ & $180.39 \pm 46.60$ & $0.000^{c)}$ \\
\hline Total cholesterol (mg/dL) & $177.48 \pm 51.3$ & $176.54 \pm 52.00$ & $180.74 \pm 48.70$ & $0.282^{c)}$ \\
\hline
\end{tabular}

Values are presented as number (\%) or mean \pm standard deviation.

BMI, body mass index; TOAST, Trial of ORG 10172 in Acute Stroke Treatment; NIHSS, National Institutes of Health Stroke Scale.

${ }^{a)}$ Chi-square test, ${ }^{\text {b) }}$ Fisher exact test, ${ }^{\text {c) }}$ independent t-test.

${ }^{*} \mathrm{p}<0.05$ (with hyperglycemia patients vs. without hyperglycemia patients).

at 12 months in patients with post-stroke hyperglycemia showed regression from $2.72 \pm 5.77$ to $3.03 \pm 6.00$ compared to that at the 6-month assessment (Table 2, Fig. 2).

\section{DISCUSSION}

Glucose concentrations in the blood are regulated 


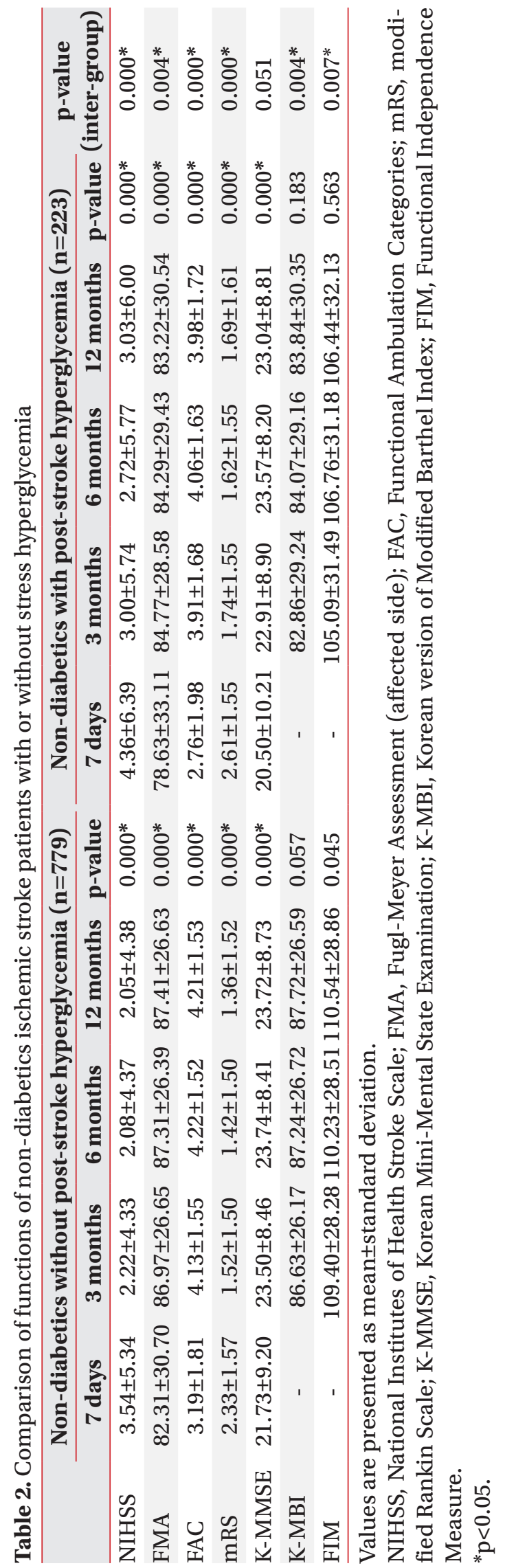

through hormonal, neural, and hepatic autoregulatory mechanisms in healthy individuals without diabetes. Illness, however, may alter normal human physiology, leading to the increased production of counter-regulatory hormones, leading to a hyperglycemic state [6]. Stress-induced hyperglycemia causes acidosis in the brain parenchyma, which negatively affects mitochondrial function in the penumbra and increases the cerebral infarct size [9]. A systematic review showed that, in patients with no history of diabetes presenting with ischemic stroke, moderately elevated glucose levels were associated with three-fold higher mortality and an increased risk of poor functional recovery [10]. Among patients with acute ischemic stroke, those with hyperglycemia upon hospital admission had a significantly higher risk of death within 30 days, 1 year, and 6 years post-stroke [26].

The limitations of previous studies include a differing definition of post-stroke hyperglycemia, thus leading to questions concerning the reliability of the results. The other limitations were the inclusion of subjects with hemorrhagic stroke as well as undiagnosed diabetes or IGT; moreover, limited outcome assessment tools were used due to a focus on mortality.

In our study, we conducted random glucose assessments at the time of admission in a consistent manner and applied reference values based on a previous study (>8 mmol/L or $>144 \mathrm{mg} / \mathrm{dL}$ ) [18]. Analysis was performed on first-time ischemic stroke patients without diabetes, excluding patients with hemorrhagic stroke. Since the two groups showed no difference in the etiological distribution according to the TOAST classification, the prognosis according to the etiology should have no effect on the outcomes. HbAlc is the most reliable parameter used exclude patients with poor glucose control; this has also been used in previous studies [10]. The enrolled patients had less severe clinical conditions and were able to participate in the serial functional assessments. None of our patients expired during the follow-up period.

A study showed that blood glucose has no effect on the amount of hemispheric brain swelling or infarct size after middle cerebral artery occlusion in rats [27]. Our study found no significant differences in the NIHSS scores at the time of admission between the two groups, indicating that post-stroke hyperglycemia does not affect the penumbra. The mean glucose-check time from onset were $4.00 \pm 4.47$ and $4.10 \pm 4.13$ hours for patients without 

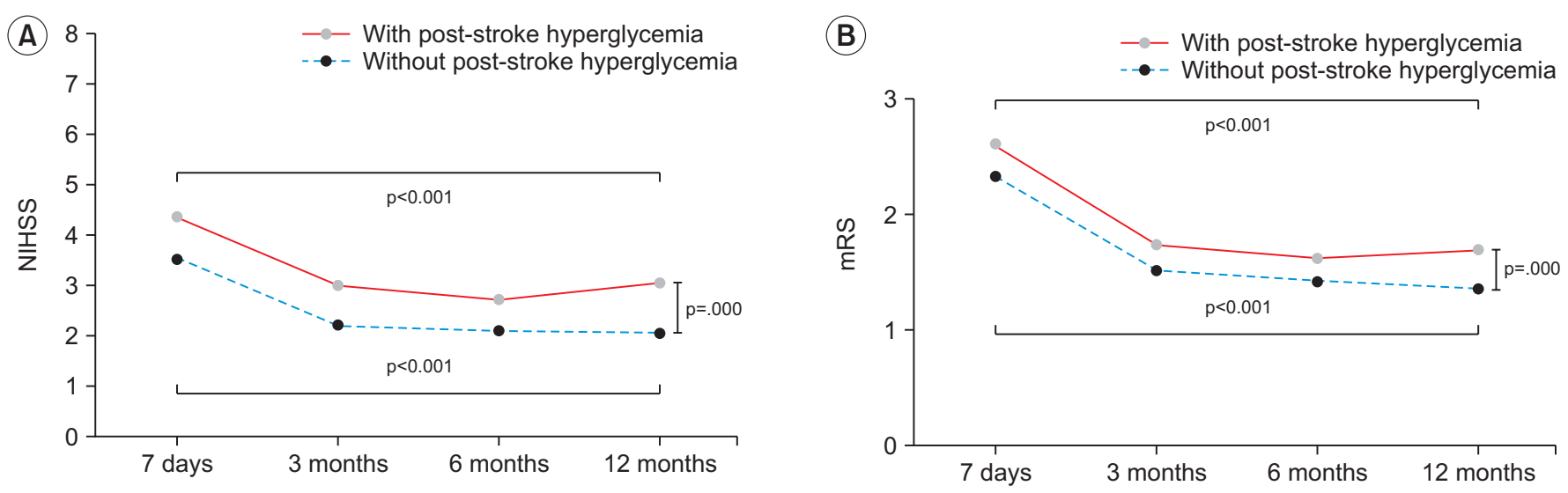

Fig. 2. Comparison of (A) National Institutes of Health Stroke Scale (NIHSS) and (B) modified Rankin Scale (mRS) between the group with or without post-stroke hyperglycemia in non-diabetic ischemic stroke patients.

and with hyperglycemia, respectively ( $\mathrm{p}>0.05)$. The importance of strict and prompt control of glucose after the onset of stroke to prevent additional brain damage is emphasized.

Patients with stress hyperglycemia showed lesser improvements in stroke severity, cognitive function, motor function, mobility and gait, and general functional assessment in the short- to long-term follow-up. Although the initial NIHSS scores showed no significant differences between the two groups $(5.17 \pm 5.56$ and $5.71 \pm 6.31$ for patients without and with hyperglycemia, respectively), it is interesting to note that the NIHSS scores showed a regression from $2.72 \pm 5.77$ to $3.03 \pm 6.00$ at 12 months in patients with post-stroke hyperglycemia compared to the values at the 6 -month assessment. The long-term functional outcomes of ischemic stroke patients without diabetes showed significant differences between the groups with or without post-stroke hyperglycemia.

Our study emphasized the long-term effects of poststroke hyperglycemia on patients' ADLs. The mechanism underlying how it increases the severity of brain injuries is already known. Although the initial NIHSS scores had no significant differences, this study proved this mechanism on long-term follow-up.

One of the limitations of our study is that the interpretation of post-stroke hyperglycemia is not fixed. Since a single-time measurement of a random glucose level at admission was used, the cut-off level used to define hyperglycemia might differ from that used in other studies, in which the fasting glucose level the morning after admission ranged from 6.1-7.8 mmol/L (110-141 mg/dL).
Patients with borderline glucose levels might have a ceiling effect [28-31].

Volumetric analysis by CT/CTA or MRI/MRA scans may be valuable to investigate and compare the severity of stroke between the groups. A study mentioned that assuming glucose was controlled after admission, monitoring for post-stroke hyperglycemia continuously may be a superior approach since it is associated with worse outcomes [32].

In conclusion, the glucose levels of ischemic stroke patients without diabetes had no significant correlation with the initial NIHSS score. The long-term effects of stress hyperglycemia showed worse functional outcomes in ischemic stroke patients without diabetes with poststroke hyperglycemia.

\section{CONFLICT OF INTEREST}

No potential conflict of interest relevant to this article was reported.

\section{ACKNOWLEDGMENTS}

This work was supported by the Research Program funded by Korea Disease Control and Prevention Agency (2019E320202).

\section{AUTHOR CONTRIBUTION}

Conceptualization: Yoon JA, Shin YI. Methodology: Yoon JA, Shin YI. Formal analysis: Yoon JA, Shin YI, Park 
MS. Funding acquisition: Kim YH. Project administration: all authors. Visualization: all authors. Writing original draft: Yoon JA, Shin YI. Writing - review and editing: Yoon JA, Shin YI. Approval of final manuscript: all authors.

\section{REFERENCES}

1. Gisselsson L, Smith ML, Siesjo BK. Hyperglycemia and focal brain ischemia. J Cereb Blood Flow Metab 1999;19:288-97.

2. Kiers L, Davis SM, Larkins R, Hopper J, Tress B, Rossiter SC, et al. Stroke topography and outcome in relation to hyperglycaemia and diabetes. J Neurol Neurosurg Psychiatry 1992;55:263-70.

3. Alvarez-Sabin J, Molina CA, Montaner J, Arenillas JF, Huertas R, Ribo M, et al. Effects of admission hyperglycemia on stroke outcome in reperfused tissue plasminogen activator: treated patients. Stroke 2003;34:1235-41.

4. Paolino AS, Garner KM. Effects of hyperglycemia on neurologic outcome in stroke patients. J Neurosci Nurs 2005;37:130-5.

5. Montori VM, Bistrian BR, McMahon MM. Hyperglycemia in acutely ill patients. JAMA 2002;288:2167-9.

6. McCowen KC, Malhotra A, Bistrian BR. Stress-induced hyperglycemia. Crit Care Clin 2001;17:107-24.

7. Weil ZM. Ischemia-induced hyperglycemia: consequences, neuroendocrine regulation, and a role for RAGE. Horm Behav 2012;62:280-5.

8. Parsons MW, Barber PA, Desmond PM, Baird TA, Darby DG, Byrnes G, et al. Acute hyperglycemia adversely affects stroke outcome: a magnetic resonance imaging and spectroscopy study. Ann Neurol 2002;52:20-8.

9. Capes SE, Hunt D, Malmberg K, Pathak P, Gerstein HC. Stress hyperglycemia and prognosis of stroke in nondiabetic and diabetic patients: a systematic overview. Stroke 2001;32:2426-32.

10. Capes SE, Hunt D, Malmberg K, Gerstein HC. Stress hyperglycaemia and increased risk of death after myocardial infarction in patients with and without diabetes: a systematic overview. Lancet 2000;355:7738.

11. Auer RN. Insulin, blood glucose levels, and ischemic brain damage. Neurology 1998;51(3 Suppl 3):S39-43.

12. Bruno A, Durkalski VL, Hall CE, Juneja R, Barsan
WG, Janis S, et al. The Stroke Hyperglycemia Insulin Network Effort (SHINE) trial protocol: a randomized, blinded, efficacy trial of standard vs. intensive hyperglycemia management in acute stroke. Int J Stroke 2014;9:246-51.

13. Yoon JA, Kim DY, Sohn MK, Lee J, Lee SG, Lee YS, et al. Effect of stress hyperglycemia and intensive rehabilitation therapy in non-diabetic hemorrhagic stroke: Korean Stroke Cohort for Functioning and Rehabilitation. Eur J Neurol 2016;23:1658-65.

14. Davidson MB. How do we diagnose diabetes and measure blood glucose control? View 1: (Diagnosing) a clinical basis for the diagnosis of diabetes. Diabetes Spectr 2001:14:67-71.

15. Goldstein LB, Adams R, Alberts MJ, Appel LJ, Brass LM, Bushnell CD, et al. Primary prevention of ischemic stroke: a guideline from the American Heart Association/American Stroke Association Stroke Council. Stroke 2006;37:1583-633.

16. Bernardini J, Callen S, Fried L, Piraino B. Inter-rater reliability and annual rescoring of the Charlson comorbidity index. Adv Perit Dial 2004;20:125-7.

17. Oh MS, Yu KH, Lee JH, Jung S, Ko IS, Shin JH, et al. Validity and reliability of a Korean version of the national institutes of health stroke scale. J Clin Neurol 2012;8:177-83.

18. Amarenco P, Bogousslavsky J, Caplan LR, Donnan GA, Hennerici MG. Classification of stroke subtypes. Cerebrovasc Dis 2009;27:493-501.

19. Gray CS, French JM, Bates D, Cartlidge NE, Venables GS, James OF. Increasing age, diabetes mellitus and recovery from stroke. Postgrad Med J 1989;65:720-4.

20. Kang Y, Na DL, Hahn S. A validity study on the Korean Mini-Mental State Examination (K-MMSE) in dementia patients. J Korean Neurol Assoc 1997;15:300-8.

21. Fugl-Meyer AR, Jaasko L, Leyman I, Olsson S, Steglind S. The post-stroke hemiplegic patient. 1: a method for evaluation of physical performance. Scand J Rehabil Med 1975;7:13-31.

22. Holden MK, Gill KM, Magliozzi MR, Nathan J, PiehlBaker L. Clinical gait assessment in the neurologically impaired: reliability and meaningfulness. Phys Ther 1984;64:35-40.

23. Burn JP. Reliability of the modified Rankin Scale. Stroke 1992;23:438.

24. Dodds TA, Martin DP, Stolov WC, Deyo RA. A valida- 
tion of the functional independence measurement and its performance among rehabilitation inpatients. Arch Phys Med Rehabil 1993;74:531-6.

25. Jung HY, Park BK, Shin HS, Kang YK, Pyun SB, Paik NJ, et al. Development of the Korean version of Modified Barthel Index (K-MBI): multi-center study for subjects with stroke. J Korean Acad Rehabil Med 2007;31:28397.

26. Williams LS, Rotich J, Qi R, Fineberg N, Espay A, Bruno A, et al. Effects of admission hyperglycemia on mortality and costs in acute ischemic stroke. Neurology 2002;59:67-71.

27. McBride DW, Matei N, Camara JR, Louis JS, Oudin G, Walker C, et al. Acute hyperglycemia does not affect brain swelling or infarction volume after middle cerebral artery occlusion in rats. Acta Neurochir Suppl 2016;121:251-5.
28. Candelise L, Landi G, Orazio EN, Boccardi E. Prognostic significance of hyperglycemia in acute stroke. Arch Neurol 1985;42:661-3.

29. Melamed E. Reactive hyperglycaemia in patients with acute stroke. J Neurol Sci 1976;29:267-75.

30. Toni D, Sacchetti ML, Argentino C, Gentile M, Cavalletti C, Frontoni M, et al. Does hyperglycaemia play a role on the outcome of acute ischaemic stroke patients? J Neurol 1992;239:382-6.

31. Lee TH, Ryu SJ, Chen ST. The prognostic value of blood glucose in patients with acute stroke. J Formos Med Assoc 1991;90:465-70.

32. Baird TA, Parsons MW, Phan T, Butcher KS, Desmond PM, Tress BM, et al. Persistent poststroke hyperglycemia is independently associated with infarct expansion and worse clinical outcome. Stroke 2003;34:220814. 